Résumés des conférences et travaux

\title{
Numismatique et diplomatique islamiques
}

\author{
Ludvik Kalus
}

\section{(2) OpenEdition Journals}

\section{Édition électronique}

URL : https://journals.openedition.org/ashp/642

DOI : 10.4000/ashp.642

ISSN : 1969-6310

\section{Éditeur}

Publications de l'École Pratique des Hautes Études

\section{Édition imprimée}

Date de publication : 1 octobre 2009

Pagination : 64-66

ISSN : 0766-0677

Référence électronique

Ludvik Kalus, " Numismatique et diplomatique islamiques », Annuaire de l'École pratique des hautes études (EPHE), Section des sciences historiques et philologiques [En ligne], 140 | 2009, mis en ligne le 06 octobre 2009, consulté le 06 juillet 2021. URL : http://journals.openedition.org/ashp/642 ; DOI : https://doi.org/10.4000/ashp.642 


\title{
NUMISMATIQUE ET DIPLOMATIQUE ISLAMIQUES
}

\author{
Directeur d'études : M. Ludvik KaLus
}

Programme de l'année 2007-2008 : I. Sultanat de Pasai (Sumatra) : sources numismatiques et épigraphiques. - II. Numismatique islamique. - III. Diplomatique islamique : documents ayyoubides et mamelouks.

\section{Sultanat de Pasai (Sumatra) : sources numismatiques et épigraphiques}

La route de la soie qui est le dénominateur commun du programme que nous poursuivons depuis plusieurs années dans nos conférences est en réalité ce qu'on appelle la route de la soie maritime, celle qui reliait la mer Rouge au monde malais se prolongeant jusqu'à la Chine. Après avoir exploré d'autres régions de l'Asie du Sud-Est touchées par cette route maritime, le directeur d'études achève actuellement, en collaboration avec Claude Guillot (CNRS, EHESS), des recherches dans la région d'Aceh (Sumatra) où se situait, de la fin du XIII ${ }^{\mathrm{e}}$ siècle au début du XVI ${ }^{\mathrm{e}}$ siècle, le sultanat musulman de Pasai. C'est là que se dirigeaient des commerçants arabes et persans pour se fournir en épices et cette région constituait une des plus importantes stations de la route de la soie maritime. Le sultanat musulman de Pasai joua un rôle primordial à deux niveaux. Tout d'abord, c'était dans le domaine international puisqu'il était l'un des nœuds les plus importants dans le commerce hauturier interasiatique et donc dans les échanges entre la Chine, l'Inde et le monde musulman central. D'autre part, ce sultanat avait une grande importance sur le plan régional puisque Pasai devint au XIII ${ }^{\mathrm{e}}$ siècle le premier État du grand archipel malais à adopter officiellement la religion musulmane, qui se répandit par la suite dans l'ensemble de l'Asie du Sud-Est insulaire.

Les documents relatifs à ce sultanat sont très rares : quelques mentions dans les histoires dynastiques chinoises, une chronique malaise recouvrant un demi-siècle, le témoignage du voyageur arabe Ibn Battûta et plus tardivement quelques rapports portugais. L'histoire de Pasai se révèle donc très malaisée à reconstituer. Mais Pasai a conservé un ensemble exceptionnel de tombes musulmanes inscrites comprenant environ 150 monuments datant du XIII ${ }^{\mathrm{e}}$ au XVI ${ }^{\mathrm{e}}$ siècle, ensemble resté jusque-là inexploité. Plusieurs prospections sur place et une longue analyse des inscriptions de ces tombes qui ont été sculptées en grande partie sur place à Sumatra mais également dans la région de Cambay dans le Goudjerate, ont permis à Claude Guillot et au directeur d'études de mettre en avant les grandes avancées dans le domaine historique que permettent ces nouvelles données, dans un ouvrage (400 p.) actuellement sous presse et intitulé Les monuments funéraires et l'histoire du sultanat de Pasai (Sumatra, Indonésie) 1297-1523. Dans cette publication, le corpus épigraphique qui constitue une inestimable source pour l'histoire du sultanat a permis d'établir la généalogie de la famille régnante et de reconstituer la société, «multinationale », du sultanat de Pasai, avec ses structures sociales. 
À Pasai, aujourd'hui petite bourgade, on trouve encore de nos jours à l'immédiat bord de mer, là où, il y a quelques années, ont été aménagés des bassins d'élevage de poissons et de crevettes (très endommagés par le tsunami du mois de décembre 2004), des tessons de céramique et de temps en temps des pièces de monnaie d'or (mais aussi d'étain) que la population se presse de porter chez des bijoutiers qui ne trouvent, hélas, rien de mieux que de les fondre pour récupérer le métal précieux.

La présence de ces pièces de monnaie est liée à l'histoire du sultanat, à l'origine puissant comptoir devenu État. Son rapide développement économique a eu comme conséquence l'émission, pour la première fois dans le monde malais, d'une monnaie d'or islamique, inscrite, certes sommairement, en arabe et portant le nom du sultan. Les premières émissions correspondaient à l'époque où l'on a installé à Pasai le descendant de l'avant-dernier calife abbasside, ceci pour donner du prestige à ce sultanat émergeant qui arrivait rapidement à son apogée. Les pièces de monnaie de Pasai portent systématiquement sur une face le surnom honorifique « malik al-zâhir » accompagné du nom du sultan régnant et sur la face opposée le surnom « al-sultân al-’âdil » qui est une sorte de garantie de la qualité de la pièce. Ce type monétaire se poursuivra encore du temps du sultanat d'Aceh qui va succéder à Pasai (jusqu'en 1579) et, modifié, il sera utilisé même après cette date.

Dans les conférences, nous avons procédé aux observations de l'évolution de ce type monétaire dans le temps, à l'aide de la publication de Ibrahim Alfian, Contribusi Samudra Pasai terhadap Studi Islam Awal di Asia Tenggara, Yogyakarta, 2005. Plus récemment, à Singapour, N. Rhodes, M. Goh Han Peng et V. Mihailovs ont entrepris une nouvelle étude consacrée à ce monnayage, étude basée sur un matériel beaucoup plus vaste. La nouvelle publication recensera le monnayage d'or du sultanat de Pasai et du sultanat d'Aceh, de la fin du XIII ${ }^{\mathrm{e}}$ siècle jusqu' au milieu du XVIII ${ }^{\mathrm{e}}$ siècle, et elle sera beaucoup plus détaillée que la précédente. Sa parution est annoncée pour 2009.

Quant aux monnaies d'étain, nous savons qu'elles existaient à Pasai déjà au milieu du XIV ${ }^{e}$ siècle puisqu'elles sont mentionnées par le célèbre voyageur maghrébin Ibn Battûta. Il semble que ces dernières ne portaient que la mention " al-sultân al-’âdil », mais une étude plus systématique reste encore à faire.

Dans les conférences, les résultats obtenus par des observations numismatiques ont été confrontés aux données fournies par des inscriptions funéraires des tombes toujours conservées à Pasai (quelque 150 tombes) dont la majorité appartient aux tombes des sultans ou de hauts dignitaires civils ou religieux. Ces inscriptions dont certaines ont été déjà analysées lors des conférences des années précédentes, contiennent des épitaphes rédigées en arabe, accompagnées de textes coraniques et de morceaux poétiques arabes exprimant des élégies. Ainsi, le corpus numismatique et épigraphique relevant du temps du sultanat de Pasai, complété par des données moins directes mentionnées plus haut, a servi d'exemple d'une utilisation directe des sources de ce type pour reconstituer l'histoire d'une entité étatique dont nous n'avions, jusqu'à maintenant, que peu de renseignements.

\section{Numismatique islamique}

Plusieurs conférences ont été consacrées à l'analyse critique d'un récent ouvrage de Jere L. Bacharach, Islamic History through Coins: an Analysis and Catalogue of 
tenth-century Ikhshidid Coinage, The American University in Cairo Press, Le Caire New York, 2006, qui a obtenu en 2007 le prix Samir Shama de la Royal Numismatic Society à Londres. Dans cet ouvrage qui est considéré comme exemplaire dans le genre, Jere L. Bacharach s'attaque au monnayage d'une petite dynastie au pouvoir en Égypte et en Palestine entre 935 et 969 . Le court règne de cette dynastie a permis à l'auteur des observations approfondies de l'évolution des types monétaires et surtout la confrontation des données numismatiques aux données tirées des chroniques contemporaines en arabe. Cette démarche - qui en soi même n'a rien d'original mais qui reste encore peu appliquée dans le cadre du monnayage islamique - rend le livre important dans la mesure où les résultats obtenus jettent une nouvelle lumière sur la relation entre les événements politiques et les différentes émissions monétaires de cette dynastie, surtout à ses débuts. Les résultats obtenus par les sources textuelle et monétaire se complètent mutuellement et souvent se confirment, jetant une lumière nouvelle sur nos connaissances de cette dynastie égyptienne d'origine turque servile. Intéressante est également l'analyse iconographique effectuée par Jere L. Bacharach, qui a pu identifier un nouveau type monétaire datant sans doute de tout début du pouvoir ikhshidide, en comparant certaines monnaies anépigraphes jusqu'à maintenant non attribuées de façon exacte aux monnaies byzantines contemporaines.

\section{Diplomatique islamique : documents ayyoubides et mamelouks}

Dans le cadre du volet «diplomatique islamique », on a procédé au déchiffrement des pétitions de l'époque mamelouke et à l'étude systématique de leur formulaire. L'écriture particulière de ces documents, difficile à déchiffrer, n'a pas découragé les auditeurs qui ont assez rapidement acquis une certaine habileté leur permettant de se concentrer davantage sur le contenu et la structure des documents. Ces documents ont été tirés de l'important article de Samuel Stern, « Petitions from the Mamlûk period (notes on the Mamluk Documents from Sinai) », dans Bulletin of the School of Oriental and African Studies, XXIX, 2 (1966), p. 23-76. 